\title{
Sugar shock: Probing Streptococcus pyogenes metabolism through bioluminescence imaging
}

\author{
Richard W. Davis IV ${ }^{1 \dagger}$, Charlotte G. Muse ${ }^{1 \dagger}$, Heather Eggleston ${ }^{1}$, Micaila Hill ${ }^{1}$, Peter \\ Panizzi $^{1}$ \\ ${ }^{1}$ Department of Drug Discovery and Development, Harrison School of Pharmacy, 720 South \\ Donahue, Auburn University, Auburn, AL 36849 \\ ${ }^{\dagger}$ contributed equally
}

Corresponding Author: Peter Panizzi, Department of Drug Discovery and Development, Auburn University, 720 South Donahue, Auburn, AL, 36849. Tel. 334-844-794; panizzi@auburn.edu.

Running title: Biosensor of Streptococcus pyogenes physiology

Keywords: bioluminescence; biosensors; pathogens; physiology; Streptococcus pyogenes; molecular imaging 
Streptococcus pyogenes (S. pyogenes) can thrive in its host during an infection, and, as a result, it must be able to respond to external stimuli and available carbon sources. The preclinical use of engineered pathogens capable of constitutive light production may provide realtime information on microbial-specific metabolic processes. Here we mapped the central metabolism of a luxABCDE-modified $S$. pyogenes Xen20 (Strep. Xen20) to its de novo synthesis of luciferase substrates as assessed by the rate of light production in response to different environmental triggers. Previous characterization predicted that the lux operon was under the myo-inositol iolE promotor. Here we show that supplementation with myoinositol generated increased Xen20 luminescence. Surprisingly, when supplemented with infection-relevant carbon sources, such as glucose or glycine, light production was diminished. This was presumably due to the scavenging of pyruvate by $L$-lactate dehydrogenase (LDH). Inhibition of LDH by its inhibitor, oxamate, partially restored luminescent signal in the presence of glucose, presumably by allowing the resulting pyruvate to proceed to acetyl-coenzyme A (CoA). This phenomenon appeared specific to the lactic acid bacterial metabolism as glucose or glycine did not reduce signal in an analogous lux $A B C D E$-modified Gram-positive pathogen, Staph. Xen29. The Strep. Xen20 cells produced light in a concentration-dependent manner, inversely related to the amount of glucose present. Taken together, our measures of microbial response could provide new information regarding the responsiveness of $S$. pyogenes metabolism to acute changes in its local environments and cellular health.

\section{Introduction}

Streptococcus pyogenes ( $S$. pyogenes) infections often manifest as necrotizing fasciitis or cellulitis (1). Surveillance Report for 2017 reported 23,650 new cases of $S$. pyogenes infections for every 33 million individuals. The incidence of cellulitis or necrotizing fasciitis over the same period was $44.5 \%$ and $4.9 \%$, respectively (2). The total rate for 2017 showed 7.6 per 100,000 cases, and $8.4 \%$ of those resulted in death (2).

An intriguing pre-clinical method for real-time in vivo tracking of these harmful pathogens during an infection is bioluminescence. Although the use of engineered light-producing microbes has limited diagnostic value, the benefit of these types of bacteria to pre-clinical experimentation is often underappreciated. For example, deposition of bacterial-targeted dyes in vivo during pre-clinical testing can be readily verified by co-localization with the light signal from one of these engineered microbes. Typically, such studies require that the parental strain be transformed with plasmids containing either the Photorhabdus luminescens lux operon cassette (luxABCDE) or the firefly luciferase (ffluc) enzyme. One significant limitation to the use of these pathogens is the gap in our understanding regarding changes in light production by these microbes. The luxABCDE cassette often is placed on a transposable element, so expression is dependent on its random insertion into the genome of the parent bacterium (3). As such, light production can be linked to cellular processes that are inherent to the microbe and report on the microbes' response to its local environments. Previously, we studied a luxABCDE-modified Streptococcus pyogenes Xen20 (Strep. Xen20) to track the spread of cutaneous infection in wild type mice (4). By ex vivo colony forming units (CFU) determination and Gram-staining histology, we noted live Streptococcus present in the distal organs of the infected animals. Our findings did not support the non-invasive bioluminescent imaging (BLI) results, suggesting either the signal was below the limit of detection or there was a breakdown of light production in vivo. We found that light production by Strep. Xen20 
decreased with increasing D-glucose concentration; thereby, essentially serving as a glucose biosensor within the animal. Recently, Mimee et al. reported on a similar lightproducing Escherichia coli (E. coli) used in an ingestible bacterial-electronic system that detects the presence of heme in a model of gastric bleeding (5). Such microbial systems or devices could serve as biosensors in venues ranging from clinical to industrial.

Light production by the emitting microbes follows the conversion of activated fatty acyl compounds to fatty aldehydes via the lux $C D E$ system (6). These fatty aldehydes drive the lux $A B$ complexes, which use molecular oxygen and reduced flavin mononucleotide $\left(\mathrm{FMNH}_{2}\right)$ to produce fatty acid, water, oxidized flavin mononucleotide, and light. Therefore, an essential requirement of bioluminescence is the availability of critical reactants, such as nonanoic, $\mathrm{FMNH}_{2}$, and adenosine triphosphate (ATP) (7). Herein, we show a differential light production based on catabolite repression and gluconeogenesis for streptococcal and staphylococcal strains of similar Lux-cassette design. Furthermore, we challenged these microbes with different stimuli (i.e., myoinositol, oxamate, and carbon sources). We monitored their relative light production to dissect processes that would promote or repress the light production pathway in these strains. Given our results, it may be possible to use these light-producing pathogens as biosensors for the rapid assessment of physiologic processes and the local microbial environment.

\section{Results}

\section{$D$-Glucose inhibits bioluminescence from Strep. Xen20}

Our previous results indicated that the luminescence of Strep. Xen20 did not accurately reflect bacterial load in vivo and suggested this may be due to a $D$-glucose- mediated inhibitory effect (4). To confirm this finding on a static medium, we plated Strep. Xen20 on Todd-Hewitt with yeast extract (THY) plates supplemented with 0 - or $50-\mathrm{mM}$ $D$-glucose. For this experiment, cells were grown overnight and imaged for luminescence (Figure 1). Colonies with $D$-glucose supplementation showed decreased luminescence. Interestingly, the presence of sheep blood (SB) also significantly reduced the bioluminescence produced by Strep. Xen20. Similar CFUs were also observed by colony counting.

\section{The lux operon in Xen20 is regulated by inositol and glucose levels}

Strep. Xen20 has the luxABCDE cassette inserted in the iol operon used for inositol catabolism. More specifically, the gene is under the iolE promoter (8), which encodes 2keto-myo-inositol dehydratase (9). The IolR repressor controls the entire iol operon (10). Under high inositol conditions, the repressor is liberated, and genes are expressed. As such, we tested the contribution of inositol to bioluminescence expression in Strep. Xen20. The results (Figure 2A) indicate increased light from bacterial cells grown in the presence of myo-inositol. In contrast, co-supplementation of Strep. Xen 20, with both inositol and a low concentration of glucose $(3 \mathrm{mM})$, overcame this inositol-dependent enhancement. All BLI signal returned to baseline in less than 3 hours (Figure 2B). To evaluate this inositol benefit in actively growing cells, equal portions of Strep. Xen 20 were spread on Luria broth (LB) plates in the absence or the presence of $6 \mathrm{mM}$ myoInositol, of $50 \mathrm{mM} D$-glucose, or both additives (Figure 2C). Results paralleled Figure 1 and the tube tests in Figure 2A-B. BLI from bacterial plates at 14, 24, and 38 hours in the presence of $D$-glucose plates showed near-complete loss of light production, and this was only slightly counteracted by the additional of myo-inositol at the 38 hour time point (Figure 2C). 
To support cross-conditional comparisons and to coordinate timing, we imaged the four distinct conditions simultaneously over two days - the Strep. Xen 20 kinetic profile showed a definite 2-reaction trace with the development of a new inositol peak at 16-17 hours that was almost $\sim 4$-fold greater than the amount of light emitted by the LB control (Figure 2D-E). Despite similar bacterial growth over the time course, the BLI signal for both the $50 \mathrm{mM} D$-glucose and $6 \mathrm{mM}$ myo-inositol supplemented conditions showed markedly lower light production. Conversely, Staph. Xen 29 showed no change in light production with $6 \mathrm{mM}$ inositol supplementation alone and an $\sim 2$-fold increase when grown in the presence of $D$-glucose that was inositol-independent (Figure 2F-G). These results agree with previous research that showed the expression of green fluorescent protein (GFP), placed under the iolE promoter, was decreased in glucose-rich conditions under the iolE promoter in Salmonella enterica serovar Typhimurium strain 14028 (11).

\section{Substrate sources for bioluminescence of Streptococcus pyogenes}

Since inositol cannot function as a carbon source in Streptococcus pyogenes M49 strains (based on the Kypto Encyclopedia of Genes and Genomes (KEGG) genomic data), we investigated the agonistic or antagonistic effects of central metabolites to the production of light. As previously stated, bacterial bioluminescence is dependent on the production of substrates by the bacterial cell (6); therefore, we investigated the link of bioluminescence to central metabolism in $S$. pyogenes (see pathway scheme in Figure 3 ). Bioluminescence is entirely dependent on the availability of activated acyl donors, which are produced de novo by the bacterial cell and serve as the substrate for the lux operon to create fatty aldehydes. These substrates are then converted to fatty acids in the presence of $\mathrm{FMNH}_{2}$ and oxygen, thereby releasing light. Activated acyl donors are synthesized in one of two ways: first, coenzyme A (CoA)-containing acyl compounds can be created via the breakdown of fatty acids by $\beta$-oxidation (green box, Figure 3); second, CoA-containing fatty acid building blocks can be created via the fatty acid biosynthesis pathway (salmon box, Figure 3 ). The second pathway is dependent upon the creation of acetyl-CoA by the glycolysis/lactic acid fermentation pathway (blue box, Figure 3).

Although the KEGG Pathways are not available for Strep. Xen20, a pathway exists for the closely related M49 serotype ancestor NZ131 (http://www.genome.jp/dbgetbin/www bget?genome:T00780) Analysis of this pathway revealed important allosteric and feedback mechanisms for bioluminescence production. First, M49 strains lack the $\beta$-oxidation path beyond the creation of hexadecanoyl-CoA from hexadentate. In contrast, $S$. aureus NCTC8325, the parental strain of a frequently manipulated strain RN4220, showed increased capacity for degradation of fatty acids. Therefore, in M49 strains, the majority of activated acyl donors must be created by the fatty acid biosynthesis pathway. Analysis of these pathways revealed M49 to have all necessary enzymes for these pathways (Supplemental Figure 2), which led us to investigate the contribution of glycolytic compounds on the BLI signal.

\section{Dependence of luminescence on glucose homeostasis}

Strep. Xen 20 processes sugars by homolactic fermentation, a process that utilizes $D$-glucose as the preferred carbon source (13). Based on KEGG genome available data, glycine is converted to pyruvate via conversion to serine by L-serine dehydratase in reactions essential to the formation of one-carbon metabolites. 
We, therefore, utilized D-glucose as a glycolytic carbon source and glycine as a nonglycolytic carbon source. $D$-Glucose is present at physiological concentrations ranging between 3.9-5 mM in healthy adult mice (14). Therefore, we tested exogenous $D$-glucose and glycine at either 3 or $6 \mathrm{mM}$ (Figure 4A). The addition of either exogenous $D$-glucose and glycine to Strep. Xen20 decreased the BLI signal. Counter to this, the addition of $D$ glucose or glycine increased luminescence expression in Staph. Xen29 (Figure 4B).

\section{Physiologically relevant limitations in bioluminescence can be attributed solely to metabolites upstream of pyruvate}

To test tissue-derived carbon sources, we perturbed the carbon source equilibrium of both light-producing pathogens (Strep. Xen20 and Staph. Xen29) by characterizing the glucose-dependency of BLI signal. The dosedependent effect of $D$-glucose on light production was monitored following incubation with M9 minimal media supplemented only with casein hydrolysate and yeast extract (Figure 5). The supplements meant to provide the microbe with amino acids and cofactors essential for protein and DNA synthesis. In this moderately enhanced M9 medium, we found that Xen20 produced detectable levels of luminescence. Providing an additional glycolytic carbon source, in the form of $D$-glucose, did not significantly increase radiance. Contrastingly, Xen29 produced only moderate levels of luminescence in the absence of further carbon sources. The addition of 6 $\mathrm{mM} D$-glucose significantly increased the production of light in Xen29. Similar to $D$ glucose, Xen20 grown in modified M9 alternatively supplemented with glycine (3 $\mathrm{mM}$ ) produced no significant increase in BLI signal. In contrast, all supplementations increased bioluminescent expression in Staph. Xen29 cells with maximal BLI signal when glycine was present (Figure 5).

\section{Restriction of luminescence is dependent on L-lactate dehydrogenase}

Homolactic fermentation produces pyruvate that is either converted to acetyl-CoA by pyruvate dehydrogenase or to lactate by Llactate dehydrogenase (blue box, Figure 3). Pyruvate conversion by pyruvate dehydrogenase would prepare substrates relevant to light production. In contrast, conversion by L-lactate dehydrogenase would scavenge pyruvate away from this potential pool. To test the effect of pyruvate conversion to lactate and the availability of the acetyl-CoA pool, various concentrations (0-64 $\mathrm{mM})$ of oxamate, an inhibitor of L-lactate dehydrogenase, were added to Strep. Xen20 cells (Figure 6) (15). However, luminescence was constant up to $32 \mathrm{mM}$ oxamate (Figure 6). At the $64 \mathrm{mM}$ oxamate, the radiance increased for the glucose-containing sample, but there was also an observed decrease in cell density. A diffusion assay tested the effect that drug eluted in to agar has on light production (Figure 6B). Equal CFUs of Strep. Xen20 cells were added to the quadrants of an agar plate, and an oxamate or relevant control discs were added (Figure 6B). Discs were then imbued with equivalent volumes applied to them $(20 \mu \mathrm{l})$, and the solutions were sterile water, glucose alone $(0.022 \mathrm{mg})$, oxamate $(0.14 \mathrm{mg})$, and both glucose and oxamate. Dried discs were placed on the agar plates. Bioluminescence production was higher in quadrants with oxamate (Figure $6 C)$.

\section{Determining plasma glucose concentrations based on relative luminescence}

Given the dependence of Xen20 bioluminescence on glucose homeostasis, we sought to correlate glucose concentration with the relative light production of Strep. Xen20. Light production of the Xen20 decreased at relatively similar rates when added to an equal 
volume of glucose in phosphate buffered saline (PBS). However, each sample displayed a different time to initial decrease and terminal luminescence (Figure 7A). Glucose concentration in the PBS and mouse plasma samples was confirmed using a commercial blood glucose meter. Based on the linear relationship, the mouse plasma had a glucose concentration of $3.3 \mathrm{mM}$ (Figure 7B). After the D-glucose standards were allowed to incubate with the Strep. Xen20 culture overnight, a exponentially decreasing relationship was found to exist between the amount of glucose and Strep. Xen20 BLI (Figure 7C). Data was analyzed using a single exponential and used to predict glucose concentration in mouse plasma. Our predicted concentration of glucose in the mouse plasma matched actual glucometer values determined independently.

\section{Discussion}

Competition for carbon sources and energy may affect the survival of Streptococcus pyogenes $(S$. pyogenes) during an infection. Previously we found that a $\operatorname{lu} x A B C D E-$ incorporated $S$. pyogenes had reduced light production in $D$-glucose enriched medium (4). Although this trait is less desirable for Strep. Xen20 utility in non-invasive imaging of infections, it highlights a unique ability of bacterial luciferase to serve as a biosensor of activated or repressed metabolic pathways. We explore the use of Strep. Xen 20 as a biosensor that could respond to changes in its local environment by altering its light production. This biosensor would fundamentally differ from the current utilization of isolated luciferases in biochemical assays. Specifically, our biosensor reports the activity of metabolic enzymes (substrates for light production) rather than on the level of promoter activity intrinsic to firefly or Renilla luciferase (under a bolus of the substrate).

Initially, genetic evidence pointed to iolE as the gene sequence interrupted by the $\operatorname{lux} A B C D E$ pathway (8). Results of inositol supplementation (Figure 2) indicate a genetic regulation of the luxABCDE bioluminescence. According to the KEGG metabolic pathways for NZ131, M49 serotype strains of Strep. Xen20 does not contain the enzymes necessary to convert this inositol further into usable carbon sources to enter glycolysis or citric acid cycle. Therefore, we tested the effects of other carbon sources to link the creation of luminescent substrates to the central metabolism of the bacterial cell.

Our analysis of the KEGG metabolic pathways for NZ131 revealed no enzymes capable of fatty acid degradation beyond the creation of hexadecanoyl-coenzyme A (see the scheme in Figure 3). Necessary activated fatty acyl compounds must then be created via the fatty acid biosynthesis pathway, which utilizes acetyl-CoA as building blocks for the creation of fatty acid chains. As previously mentioned, acetyl-CoA is produced in $S$. pyogenes via homolactic fermentation. Taken together, this suggests $S$. pyogenes has a more simplified route to the production of the essential lightproducing building block than its counterpart, S. aureus.

Glucose is utilized in one of two ways: first, it can be transported into the cell by phosphotransferases, which convert it to glucose-6-phosphate during uptake, and fed into the fermentation process; second, it can be converted to glucose-6-phosphate by hexokinase (13). The addition of $D$-glucose and glycine were shown here to decrease the bioluminescence of Strep. Xen20. As seen in Figure 3, and reviewed elsewhere (13), excess glucose during homolactic fermentation causes the conversion of pyruvate to lactate by the enzyme L-lactate dehydrogenase (LDH) due to increased levels of fructose-bisphosphate (FBP), intensified by the inhibition of pyruvate conversion to acetyl-CoA by increased triose phosphate levels. Therefore, the decreased 
bioluminescence observed in $D$-glucose supplementation may be due to the reduced acetyl-CoA pool, and subsequent lack of fatty acid biosynthesis precursors. As such, we tested the addition of an inhibitor of $\mathrm{LDH}$ to attempt to restore the acetyl-CoA pool and improve the luminescent signal (15). Oxamatecontaining discs allowed for the increased light production in the dispersion area (Figure 6). Therefore, bioluminescence is diminished in the presence of increased glucose due at least in part to its generation of FBP. This FBP acts on LDH to scavenge the pool of available pyruvate, committing it to the production of lactate rather than the fatty acyl precursor acetyl-CoA. Glycine supplementation showed comparable bioluminescence levels at concentrations equal to those of $D$-glucose. Although glycine is first converted to pyruvate, gluconeogenesis can convert this pyruvate back to triose phosphate and/or FBP, creating a similar effect to $D$-glucose supplementation.

Interestingly, the addition of amino acid sources, such as casein hydrolysate, relaxed the inhibition of bioluminescent signal by $D$ glucose and glycine supplementation (Figure 5). According to the KEGG diagram for $S$. pyogenes NZ131, L-alanine can be converted to pyruvate and contribute to luminescence. In contrast, amino acids that are converted to fumarate are unable to contribute due to the lack of citrate cycle activity. Leucine, valine, and isoleucine are converted to their subsequent oxopentanoates, but subsequent reactions are not possible due to a lack of 3methyl-2-oxobutanoate dehydrogenase. It is unclear which amino acid or a combination of amino acids plays a role in increasing the Strep. Xen20 light production in the presence of glucose and, as such, dissection of that would require further study.

The application of the data gathered here led us to investigate how Strep. Xen20 might be used as a living sensor of local glucose concentration. The results of our glucose standards and mouse plasma glucose levels give proof-of-concept evidence that Strep. Xen20 can be used to determine the approximate levels of blood glucose in a given sample, similar to the heme sensing capabilities of the luminescent E. coli. (5) Here, we evaluated the Strep. Xen20 luminescence using mouse plasma. However, it is entirely feasible that Strep. Xen20 could detect glucose in other species and in the host.

To our knowledge, these results are the first to highlight the effects of the central metabolism on the bacterial luciferase system. It is conceivable that directed insertion of the luxABCDE operon could generate a biosensor that reporters on the presence of trace heavy metals or the presence of toxicity compounds (such as arsenic). It would be easy to ignore the complications of light production by using nonintegrated plasmid versions of LuxAB in these pathogens and injection luciferin substrate, such as the ffluc system previously described in $S$. pyogenes (16). This would be simpler, in many regards, but future correlations of metabolism-dependent light production with RNA-seq technology could provide new avenues for the high-throughput assessment of compounds that disrupt molecular pathways regulating these dangerous pathogens. These would help identify non-redundant processes in these bacteria that if targets would not adversely affect human/host metabolism.

\section{Experimental Procedures}

Chemicals and Reagents - Brain-Heart Infusion (BHI), Sheep Blood Agar (SBA), and Todd-Hewitt with yeast extract (THY) were from BD Biosciences (San Jose, CA) or RPI. Kanamycin and $D$-glucose were purchased from Research Products International (RPI; Mt. Prospect, IL). Glycine was purchased from AMERSCO (Solon, OH). Unless otherwise specified, all reagents were purchased from 
Sigma Aldrich. M9 minimal medium was prepared as previously described (17).

Bacterial Strains, Cultivation Conditions, and Imaging - Strains Strep. Xen20 and Staph. Xen29 (PerkinElmer Inc., Waltham, MA) were grown to stationary phase $\left(\mathrm{OD}_{600 \mathrm{~nm}}>1\right)$ in $\mathrm{BHI}$ broth for 18 hours at $37{ }^{\circ} \mathrm{C}$ and approximate concentration determined by light scattering at $\mathrm{OD}_{600 \mathrm{~nm}}$ per manufacturer instructions. Cultivation conditions were altered either in the liquid media or on solid media and supplemented with additive, as indicated. Plates and tubes were imaged on the IVIS Lumina XRMS or Lumina II system (PerkinElmer Inc.). BLI is reported as calibrated units of radiance $(\mathrm{p} / \mathrm{sec} / \mathrm{cm} 2 / \mathrm{sr})$ in LivingImage software version 4.7 (PerkinElmer Inc.), allowing for comparisons between detectors.

Bioluminescence Kinetic Assays - To test if the lux operon was indeed under the inositol promoter (iolE) (8), we diluted bacteria in PBS containing myo-inositol, D-glucose, or both in a final $1 \mathrm{~mL}$ volume at equivalent $\mathrm{OD}_{600 \mathrm{~nm}}$. Tubes were incubated and imaged in the IVIS Lumina XRMS on a $37{ }^{\circ} \mathrm{C}$ stage and imaged every 20-minutes for 160 minutes. Strep. Xen20 was also streaked on a secondary set of plates with Luria broth (LB, RPI) alone or supplemented with either $6 \mathrm{mM}$ myo-inositol, $50 \mathrm{mM} D$-glucose, or both myo-inositol and $D$ glucose. Plates were incubated at $37^{\circ} \mathrm{C}$ and imaged at 14, 24, and 38 hours. Catabolite repression was assessed by supplementation of agar with myo-inositol or $D$-glucose or both in 6-well, flat-bottomed culture plates (Costar, Corning, NY) and imaged every 1.5 hours with the IVIS Lumina II for 48 hours.

The effect of carbon source availability was determined by incubating cells in sterile PBS (control) or PBS-supplemented with either 3 $\mathrm{mM}$ or $6 \mathrm{mM}$ of $D$-glucose or glycine. Sample tubes were diluted and imaged as before. To determine the contribution of growth rate to Strep. Xen20 expression, this process was repeated for M9 minimal medium supplemented with $1 \%$ casein hydrolysate and $0.3 \%$ yeast extract. This media provides necessary cofactors for growth in the absence of confounding carbon sources.

Oxamate Inhibition Assay - To determine the contribution of L-lactate dehydrogenase on Strep. Xen20 bioluminescence, Strep. Xen20 cells in the stationary phase were added to PBS containing a final concentration of $6 \mathrm{mM} D$ glucose in a 96-well plate. Varying amounts of oxamate were added for final concentrations of 0-256 mM. $\mathrm{OD}_{600 \mathrm{~nm}}$ and luminescence were measured using a Thermo Fischer VarioSkan plate at 20-minute intervals over 160 minutes at a constant $37^{\circ} \mathrm{C}$. For comparison purposes, cells treated with oxamate were compared to those untreated in both the control and glucosesupplemented groups. In addition, Strep. Xen20 was grown and imaged for bioluminescence over 48 hours on an LB agar plate containing discs saturated with $20 \mathrm{mcL}$ of solutions containing $6 \mathrm{mM}$ oxamate, $6 \mathrm{mM} D$ glucose, or both. The discs were prepared by the addition of 4 equal volume solutions $(20 \mu \mathrm{l})$ corresponding to sterile water, glucose alone $(0.022 \mathrm{mg})$, oxamate $(0.14 \mathrm{mg})$, and both glucose and oxamate. The discs were dried before placing them on the agar plates.

\section{Plasma Glucose Effect on Bioluminescence -}

To evaluate Strep. Xen20 as a potential monitor of local plasma glucose concentrations, Strep. Xen20 grown to the stationary phase in BHI was combined in a 1:1 ratio with glucose in PBS at concentrations between 0 and $10 \mathrm{mM}$. Strep. Xen20 was also combined with C57BL6 mouse plasma (Innovative Research, Novi, $\mathrm{MI})$. The light production was then monitored at 20-minute intervals at $37{ }^{\circ} \mathrm{C}$ for 2 hours using the Glomax 20/20 Luminometer (Promega, Madison, WI). A final measurement was also taken after the samples had been 
allowed to incubate overnight at room temperature. The glucose level in the glucose standards and mouse plasma were also measured directly using a commercially available ReliOn PRIME blood glucose meter (Wal-Mart Stores, Inc. Bentonville, AR) ReliOn ${ }^{\mathrm{TM}}$ PRIME blood glucose test strips. The meter was unable to determine the glucose levels at $<2 \mathrm{mM}$.

Statistical Analysis - Statistical means of each group were analyzed using two-way, repeated- measures analysis of variance (ANOVA) on $\mathrm{R}$ 3.1.2 (http://www.r-statistics.com/on/r/). Time, substrate, and the interaction of time* substrate were analyzed for each medium and each bacterium. Results were reported for statistical values $p<0.05$, or no significant difference for $\mathrm{p}>0.05$.

Data availability - All remaining data are contained within the article and Supporting Information 
Biosensor of Streptococcus pyogenes physiology

Funding and additional information This research was supported in part by NIH R01HL114477 to PRP. The content of the paper is solely the responsibility of the authors and does not necessarily represent the official views of the National Institutes of Health.

Conflict of Interest: The authors declare no conflicts of interest in regards to this manuscript.

\section{References}

1. Carapetis, J. R., Steer, A. C., Mulholland, E. K., and Weber, M. (2005) The global burden of group A streptococcal diseases. The Lancet infectious diseases 5, 685-694

2. (2013) Centers for Disease Control and Prevention. 2013. Active Bacterial Core Surveillance Report, Emerging Infections Program Network, Group A Streptococcus, 2013. . Available via the Internet: http://www.cdc.gov/abcs/reportsfindings/survreports/gas13.pdf\}.

3. Francis, K. P., Yu, J., Bellinger-Kawahara, C., Joh, D., Hawkinson, M. J., Xiao, G., Purchio, T. F., Caparon, M. G., Lipsitch, M., and Contag, P. R. (2001) Visualizing pneumococcal infections in the lungs of live mice using bioluminescent Streptococcus pneumoniae transformed with a novel gram-positive lux transposon. Infection and immunity 69, 3350-3358

4. Davis, R. W. t., Eggleston, H., Johnson, F., Nahrendorf, M., Bock, P. E., Peterson, T., and Panizzi, P. (2015) In Vivo Tracking of Streptococcal Infections of Subcutaneous Origin in a Murine Model. Mol Imaging Biol

5. Mimee, M., Nadeau, P., Hayward, A., Carim, S., Flanagan, S., Jerger, L., Collins, J., McDonnell, S., Swartwout, R., Citorik, R. J., Bulovic, V., Langer, R., Traverso, G., Chandrakasan, A. P., and Lu, T. K. (2018) An ingestible bacterial-electronic system to monitor gastrointestinal health. Science 360, 915-918

6. Meighen, E. A. (1993) Bacterial bioluminescence: organization, regulation, and application of the lux genes. FASEB journal : official publication of the Federation of American Societies for Experimental Biology 7, 1016-1022

7. Meighen, E. A. (1991) Molecular biology of bacterial bioluminescence. Microbiological reviews 55, 123-142

8. Park, H. S., Francis, K. P., Yu, J., and Cleary, P. P. (2003) Membranous cells in nasal-associated lymphoid tissue: a portal of entry for the respiratory mucosal pathogen group A streptococcus. Journal of immunology 171, 2532-2537

9. Yoshida, K., Yamaguchi, M., Ikeda, H., Omae, K., Tsurusaki, K., and Fujita, Y. (2004) The fifth gene of the iol operon of Bacillus subtilis, iolE, encodes 2-keto-myo-inositol dehydratase. Microbiology 150, 571-580

10. Yoshida, K. I., Shibayama, T., Aoyama, D., and Fujita, Y. (1999) Interaction of a repressor and its binding sites for regulation of the Bacillus subtilis iol divergon. Journal of molecular biology 285, 917-929

11. Kroger, C., Srikumar, S., Ellwart, J., and Fuchs, T. M. (2011) Bistability in myo-inositol utilization by Salmonella enterica serovar Typhimurium. Journal of bacteriology 193, 1427-1435

12. McShan, W. M., Ferretti, J. J., Karasawa, T., Suvorov, A. N., Lin, S., Qin, B., Jia, H., Kenton, S., Najar, F., Wu, H., Scott, J., Roe, B. A., and Savic, D. J. (2008) Genome sequence of a nephritogenic and highly transformable M49 strain of Streptococcus pyogenes. Journal of bacteriology 190, 7773-7785

13. Levering, J., Musters, M. W., Bekker, M., Bellomo, D., Fiedler, T., de Vos, W. M., Hugenholtz, J., Kreikemeyer, B., Kummer, U., and Teusink, B. (2012) Role of phosphate in the central metabolism of two lactic acid bacteria--a comparative systems biology approach. The FEBS journal 279, 1274-1290 
Biosensor of Streptococcus pyogenes physiology

14. Lee, S. M., and Bressler, R. (1981) Prevention of diabetic nephropathy by diet control in the $\mathrm{db} / \mathrm{db}$ mouse. Diabetes 30, 106-111

15. Feldman-Salit, A., Hering, S., Messiha, H. L., Veith, N., Cojocaru, V., Sieg, A., Westerhoff, H. V., Kreikemeyer, B., Wade, R. C., and Fiedler, T. (2013) Regulation of the activity of lactate dehydrogenases from four lactic acid bacteria. The Journal of biological chemistry 288, 2129521306

16. Loh, J. M., and Proft, T. (2013) Toxin-antitoxin-stabilized reporter plasmids for biophotonic imaging of Group A streptococcus. Applied microbiology and biotechnology 97, 9737-9745

17. (2010) M9 minimal medium (standard). Cold Spring Harbor Protocols

\section{Footnotes}

Abbreviations are: S. pyogenes, Streptococcus pyogenes; luxABCDE, Photorhabdus luminescens lux operon cassette; ffluc, firefly luciferase; Strep. Xen20, Streptococcus pyogenes Xen20; E. coli, Escherichia coli; $\mathrm{FMNH}_{2}$, reduced flavin mononucleotide; THY, Todd-Hewitt with yeast extract; SB, sheep blood; GFP, green fluorescent protein; KEGG, Kypto Encyclopedia of Genes and Genomes; CoA, coenzyme A; BHI, Brain-Heart Infusion; SBA, Sheep Blood Agar (SBA); ANOVA, analysis of variance; LB, Luria broth; PBS, phosphate buffer saline; NAD ${ }^{+}$, nicotinamide adenine dinucleotide; triose-P, triose phosphate; $\mathrm{HPr}$, histidine-containing carrier protein; HPr (HPr(Ser-P); PEP, phosphoenolpyruvate; BPG, 1,3-bisphosphoglyceric acid 
bioRxiv preprint doi: https://doi.org/10.1101/2022.01.15.476453; this version posted January 16, 2022. The copyright holder for this preprint (which was not certified by peer review) is the author/funder. All rights reserved. No reuse allowed without permission.

Biosensor of Streptococcus pyogenes physiology

\section{Figures}
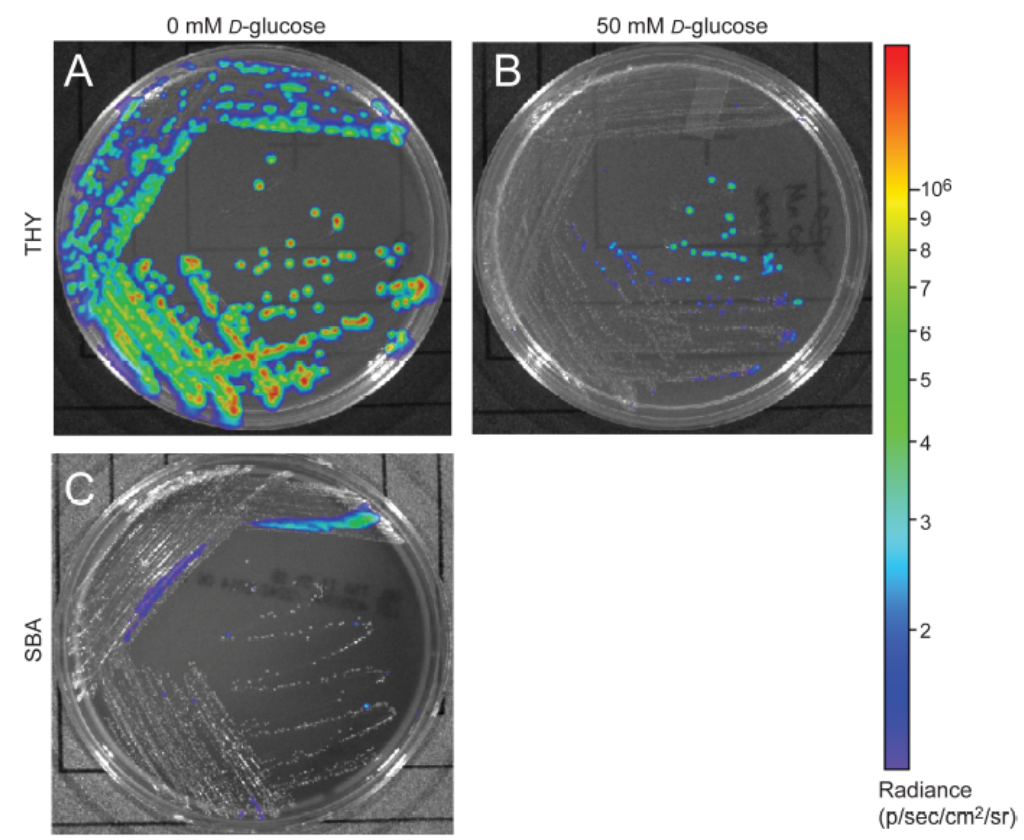

Figure 1. Glucose effect on bioluminescent production of $\boldsymbol{S}$. pyogenes Xen20. Strep. Xen20 was plated on Todd Hewitt with yeast extract (THY) without (Panel A) or with the addition of excess D-glucose (Panel B) or on sheep blood agar (SBA) and incubated for 20 hours at 37 C. 
A

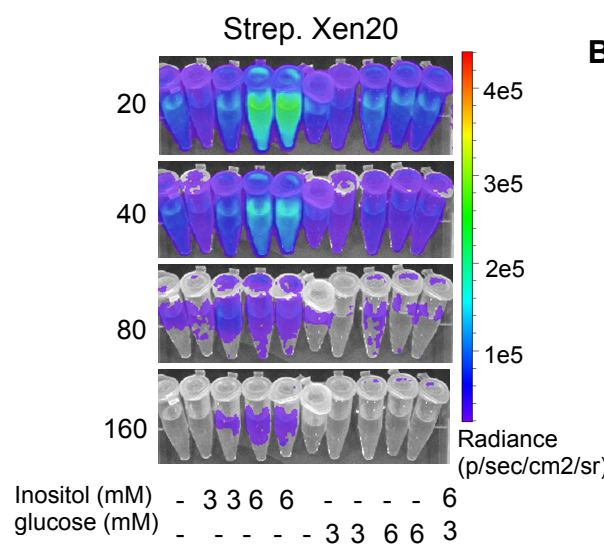

D

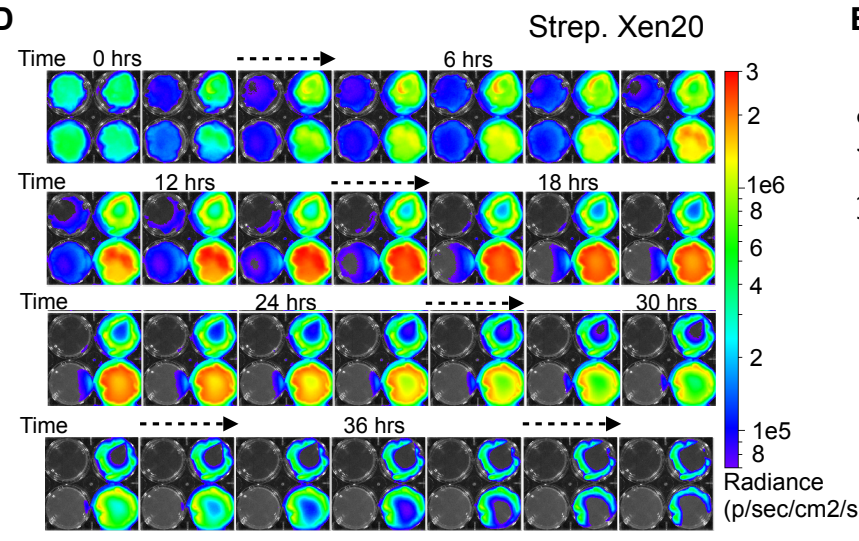

$\mathbf{F}$

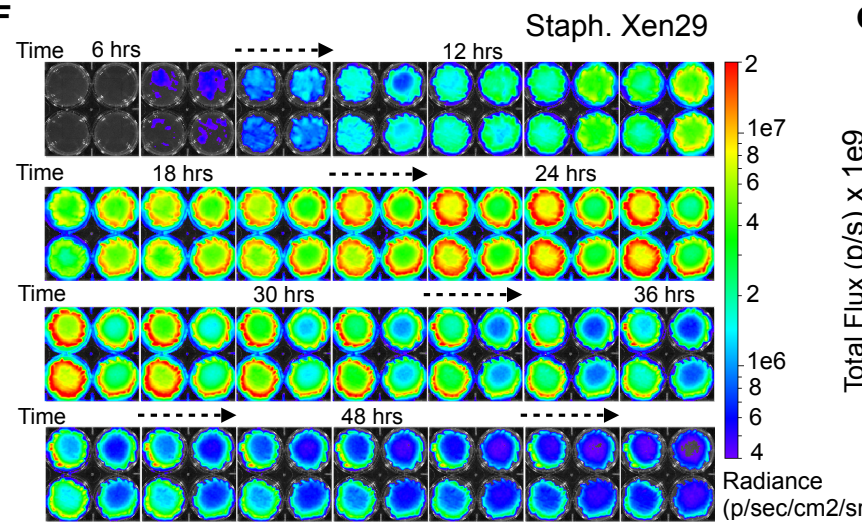

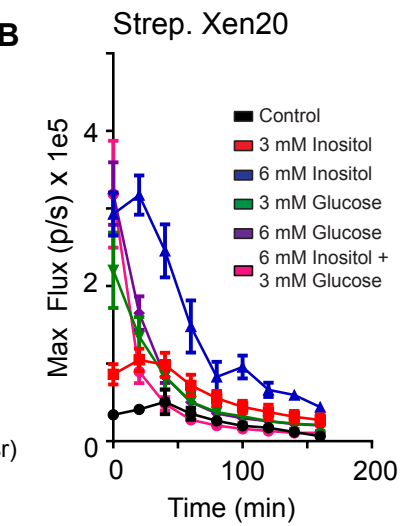

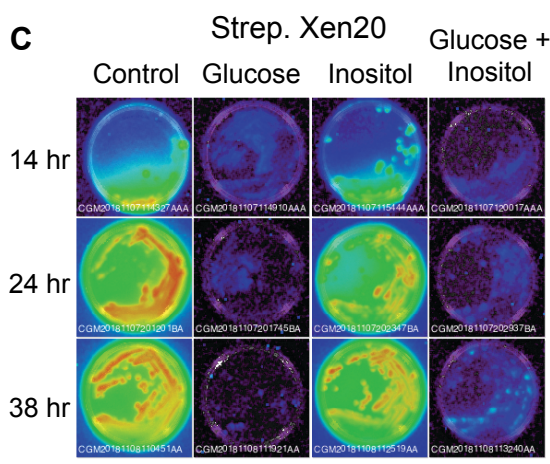

E Strep. Xen20
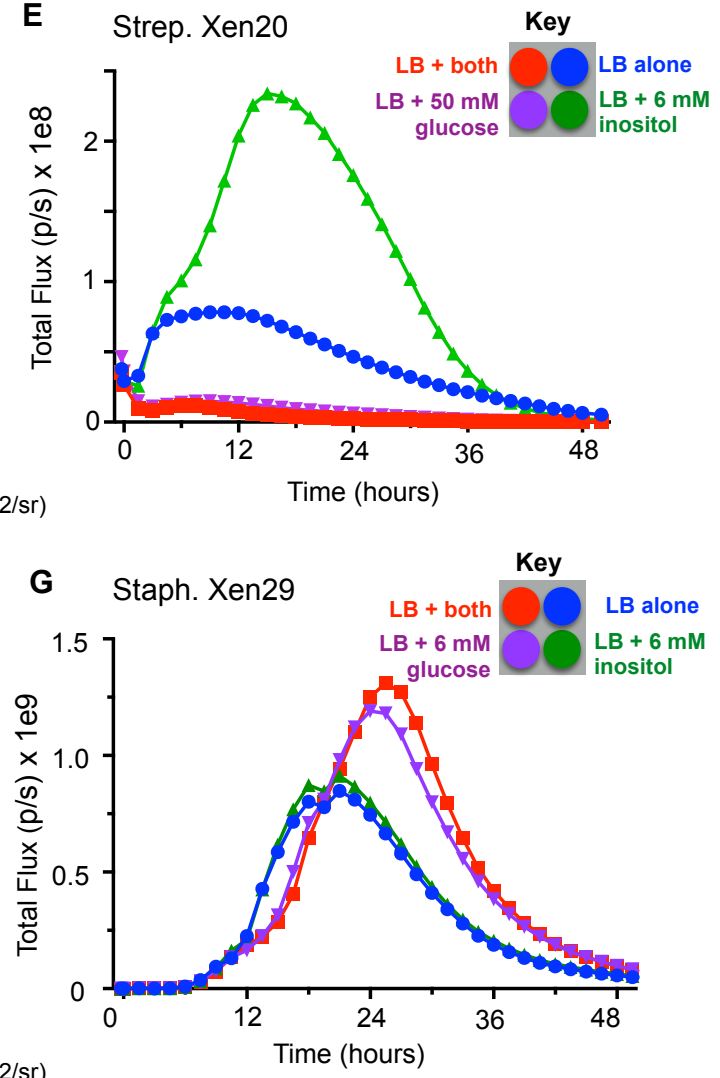

Figure 2. Myo-inositol regulates luminescence of $\boldsymbol{S}$. pyogenes Xen20. $A$. Representative time course of Strep. Xen20 cells. Samples were grown to stationary phase and then diluted in fresh PBS supplemented with one of either 3 or $6 \mathrm{mM}$ myo-inositol or 3 or $6 \mathrm{mM} D$-glucose. $B$. Quantification of luminescent signals from samples shown in Panel A shown as the maximum rate of photons generated (Max flux). C. BLI of $65 \mathrm{~mm}$ LB plates containing no additive (control), 50mM glucose, $6 \mathrm{mM}$ myo-inositol, or both taken at 14, 24, and 38 hours after incubating at 37 C. D. Strep. Xen 20 growth on LB plates containing either no additive (control), $50 \mathrm{mM}$ glucose, $6 \mathrm{mM}$ myo-Inositol, or both and imaged over 48 hours in the IVIS. E. BLI quantification of the plates from Panel $D$ as the total rate of photons generates (Total flux) $F$. Staph. Xen29 incubated on LB plates containing either no additive (control), 50mM glucose, 
bioRxiv preprint doi: https://doi.org/10.1101/2022.01.15.476453; this version posted January 16, 2022. The copyright holder for this preprint (which was not certified by peer review) is the author/funder. All rights reserved. No reuse allowed without permission.

Biosensor of Streptococcus pyogenes physiology

$6 \mathrm{mM}$ myo-Inositol, or both and imaged over 60 hours in the IVIS. G. BLI quantification of the plates from Panel $D$ as the total rate of photons generates (Total flux). 


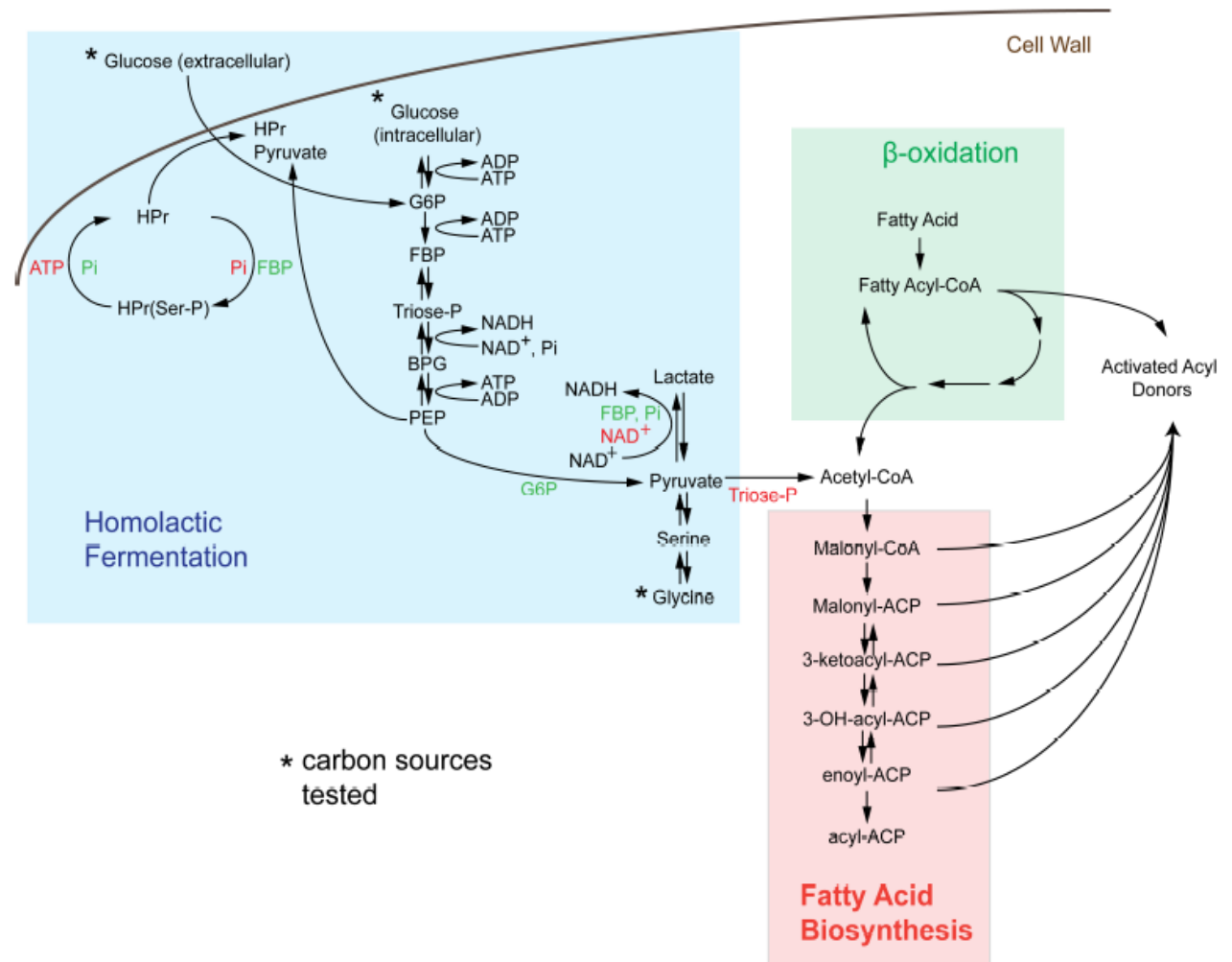

Figure 3. De novo synthesis of acyl donors via central carbon metabolism in Streptococcus. Activated acyl donors, the substrates for the luxABCDE machinery, are made by the breakdown of fatty acids through $\beta$-oxidation (green region) or by the synthesis of an activated carrier protein $(A C P)$-containing metabolites via fatty acid biosynthesis (salmon region). Since $S$. pyogenes lacks the $\beta$-oxidation pathway, all substrates for light production in Strep. Xen 20 are created by acetyl-coenzyme A (CoA)-dependent fatty acid biosynthesis generated by homolactic fermentation (blue box). The asterisk indicates the sole carbon sources tested. Allosteric activators, such as inorganic phosphate $(P i)$, fructose bisphosphate $(F B P)$, and glucose-6phosphate $(G 6 P)$ are shown in green text, and allosteric inhibitors, namely adenosine triphosphate $(A T P)$, nicotinamide adenine dinucleotide $\left(N A D^{+}\right)$, and triose phosphate (Triose- $P$ ) are shown in red text. Abbreviation used in here is as following: histidine-containing carrier protein $(H P r)$, phosphorylated $\operatorname{HPr}(H \operatorname{Pr}(\operatorname{Ser}-P))$, phosphoenolpyruvate $(P E P)$, and 1,3bisphosphoglyceric acid $(B P G)$. 

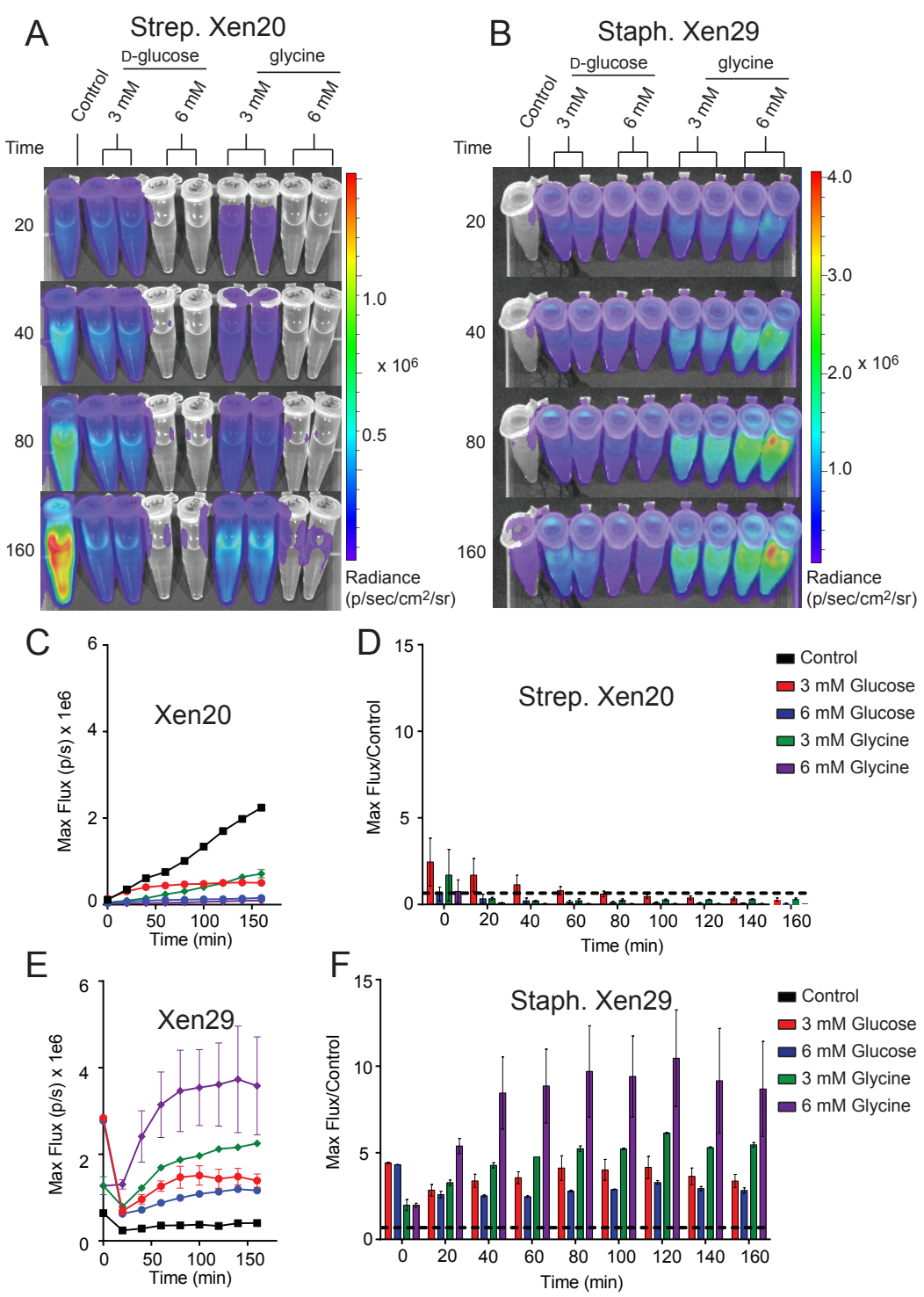

Figure 4. The effect of monosaccharides on bioluminescence production by Gram-positive pathogen. A. Representative time course of Strep. Xen20 or Staph. Xen29. For all samples, cells were grown to stationary phase then diluted in fresh phosphate-buffered saline (Control) supplemented with either $D$-glucose or glycine as indicated. B. Similar time course for Xen29 $(S$. aureus). C. Quantification of the Strep. Xen20 luminescent signal from Panel A. C-D. Quantification of luminescent signal from samples shown in Panel A for both Strep. Xen20 and Staph. Xen29 pathogens represented as either the maximum rate of photons generated (shown on the left as Max flux) or as the relative maximum signal normalized to the control (shown on the right as Flux/Control) displayed as a function of time. The black dashed line indicates a ratio at the threshold of 1.0. E-F. Similar quantification of Panel B for Staph. Xen29. All samples are 
bioRxiv preprint doi: https://doi.org/10.1101/2022.01.15.476453; this version posted January 16, 2022. The copyright holder for this preprint (which was not certified by peer review) is the author/funder. All rights reserved. No reuse allowed without permission.

Biosensor of Streptococcus pyogenes physiology

color coded as indicated and the experiment performed, as shown in the Materials and Methods section. 

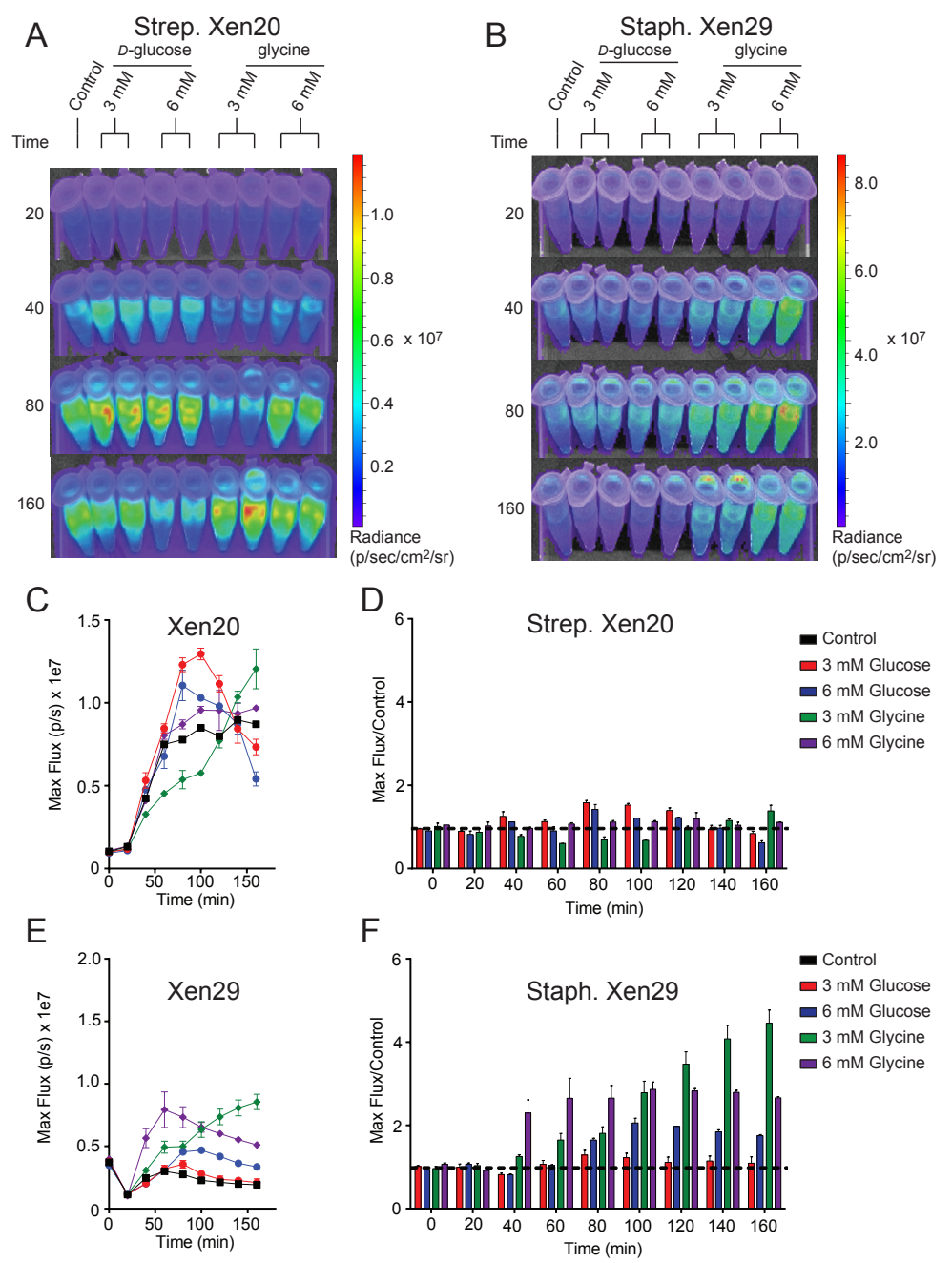

Figure 5. Increased luminescence production by Gram-positive pathogen incubated in M9 medium supplemented with casein hydrolysate and yeast extract. $\mathrm{OD}_{600 \mathrm{~nm}}$ and luminescence were measured using a Thermo Fischer VarioSkan plate at 20-minute intervals over 160 minutes at a constant $37^{\circ} \mathrm{C}$. A. Representative time course of Strep. Xen20. For all samples, cells were grown to stationary phase then diluted in fresh M9 medium containing casein hydrolysate and yeast extract (control) supplemented with either $D$-glucose or glycine as indicated. $B$. Similar time course for Xen29 (S. aureus). C. Quantification of the Strep. Xen20 luminescent signal from Panel A. $C-D$. Quantification of the Strep. Xen20 luminescent signal from Panel A represented as either the maximum rate of photons per second (Max flux) and as the relative maximum signal normalized to the control (shown on the right as Flux/Control) displayed as a function of time. The black dashed line indicates a ratio at the threshold of 1.0.E-F. Similar quantification of Panel B for Staph. Xen29. All samples are color coded as indicated and the experiment performed, as shown in the Materials and Methods section. 

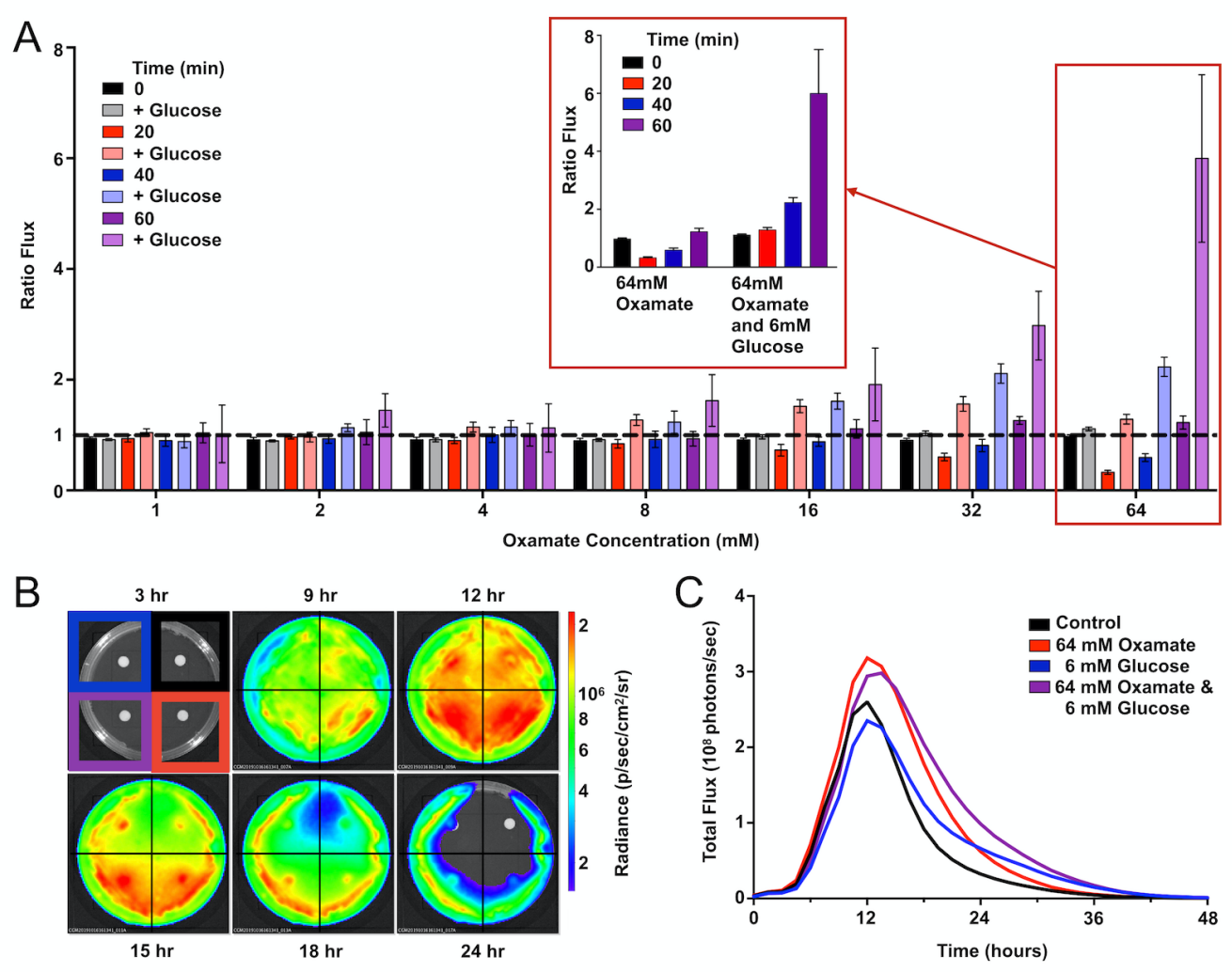

Figure 6. Oxamate inhibition partially recovers the bioluminescent signal. A. Strep. Xen20 in the stationary phase were combined with PBS alone (control) or PBS with concentrations of 6 $\mathrm{mM}$ glucose and 0-64 mM oxamate. Represented is the flux from cells treated with oxamate divided by the flux from cells in PBS alone (Ratio Flux). The data represents seven trials without or without glucose at the indicated times. Expansion of the $64 \mathrm{mM}$ oxamate concentration with and without glucose over 60 minutes shown (red inset). B. Strep. Xen20 was grown on LB agar with disks containing either a water control (upper right), $64 \mathrm{mM}$ oxamate (lower right), $6 \mathrm{mM}$ glucose (upper left), or both glucose and oxamate (lower left) monitored for 48 hours. $C$. Quantification of the luminescent signal from Panel $\mathrm{C}$ as the total rate of photons generates (Total flux). 
A

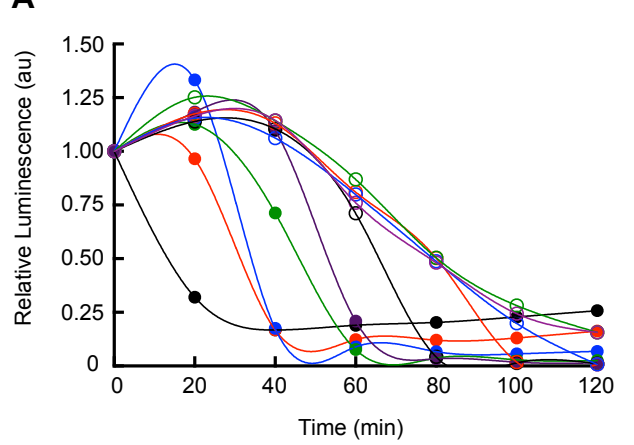

B
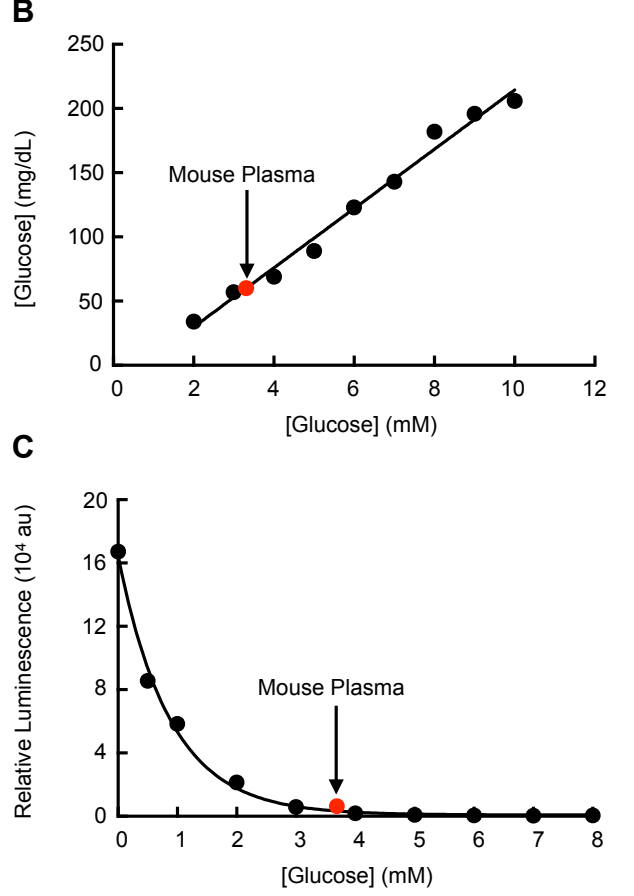

Figure 7. Utilizing Strep. Xen20 bioluminescence production to estimate plasma glucose concentrations. A. Strep. Xen20 cells in the stationary phase were added to equal volumes of PBS with 0-10 $\mathrm{mM}$ glucose. The luminescence was measured with a luminometer every 20 minutes for 2 hours. $B$. The glucoses level $(\mathrm{mg} / \mathrm{dL})$ of each PBS and glucose solution was measured using a commercially available blood glucose meter. Mouse plasma (indicated on graph in red) was also tested for blood glucose levels, which was used to estimate millimolar concentration. $C$. Final luminescence in the Strep. Xen20 sample in glucose between 0 and $8 \mathrm{mM}$ after incubation overnight. The exponential relationship yielded an equation of $y=167000 \mathrm{e}^{-0.882 \mathrm{x}}$ $\left(\mathrm{R}^{2}=0.9861\right)$ and was used to estimate the mouse plasma glucose level based on luminescence of the Strep. Xen20. 